\title{
LESSON 37
}

MARGINS: Pica, 20-70; Elite, Type this sentence as many times as your teacher asks.

$30-80$

Warm up

The quotation mark key (")

Leave no space after an initial mark or before a final mark. Punctuation at the end may be inside or outside quotation marks as long as it is consistent.

The brackets ( key

Leave one space before the ( but no space after it.

The brackets ) key

Leave no space before / but leave one space after it, except before punctuation.

Clinic

4 and 7

3 and 8

2 and 9

5 and 6

All numbers

The \% key

Use the ; finger to type the \% key after depressing the left shift key. If the key is not on the bottom row, your teacher will give you the first line of the drill.

Test your theory

Use double-line spacing.

Make your own right-hand line endings.

UNIT 10/CONSOLIDATION
1. The bank raiders quickly passed the cash into a taxi and joined the fight which may have stopped their escape through the maze of streets.

Type each line 3 times. Keep your eyes on the copy.

2. sss sws sw2 sw" sw" s"s 2 "2 "2" s"s sw"f sw"f "2"s

3. "Come," he said, "follow me to the park gate now."

4. "That is all," she said, "now you may go outside."

Type this line 3 times.

5. Ill lo9 lol lol lll llo llo lolj lolj lol llol lol Type each line 3 times. Keep your eyes on the copy.

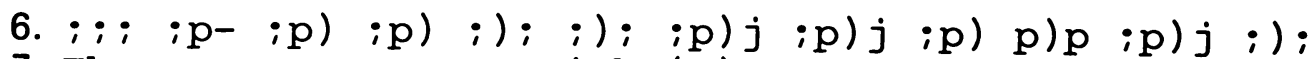
7. The scores were Ipswich (3) Nottingham Forest (i).

Type each line 3 times paying attention to good typing technique.
8. fr4 fr4 444 f $4 r$ f $44 f$ fr4 ju7 ju7 777 j7j j77j ju7j
9. de3 de3 d3d 333 d 3e3 d3d ki8 ki8 k8k k88k 888k k8k
10. sw2 sw2 s2s s2s 222s s2s 109 lo9 19119999911091
11. ft5 ft5 f5f 555 ft5t f5f ju6 ju6 666 j6j jy6y jy6j
12. The 129 children paid $£ 358$ to go on the day visit.
13. There were only 14 days left to make the 670 bags.
14. The sun shone for 295 hours in the 31 day session.

Do not leave a space before the \% sign but leave one space after it, except before punctuation.

15. $i ; i \quad i \frac{1}{2} ; \quad i \frac{1}{2} ; \quad ; \% ; \quad ; \% ; \quad i \% ; j \quad ; \% ; \quad ; \% ; \quad ; \% \frac{1}{2} \% \quad ; \% ; \quad ; \% \frac{1}{2} \% \quad ; \% ;$

16. Take $25 \%$ off the paint and $15 \%$ off the wall-paper.

17. Give $30 \%$ for profit and a further $10 \%$ for the tax.

Put in the missing words as you type these sentences.
1. A sheet of $A 4$ paper is
$\mathrm{x} \mathrm{mm}$.
2. A sheet of A5 paper is $x \quad \mathrm{~mm}$.
3. There are spaces in $25 \mathrm{~mm}$ of Pica type.
4. There are spaces in $25 \mathrm{~mm}$ of Elite type.
5. There are lines down a sheet of $A 4$ paper.
6. space/s are left after a full stop at the end of a sentence.
7. space/s are left after a question mark.
8. $\quad$ space/s are left after a comma.
9. When underscoring you the final punctuation mark.
10. The abbreviation Messrs. stands for

\section{LESSON 37}

